\title{
Influence of regular soccer or swimming practice on gross motor development in childhood
}

\author{
Helena A. Rocha ${ }^{1}$, Daniel A. Marinho', ${ }^{1,2}$ Boris Jidovtseff ${ }^{3}$, António J. Silva ${ }^{2,4}$, Aldo M. \\ Costa $1,2,5^{*}$
}

ORIGINAL ARTICLE

\begin{abstract}
The objective of this study was to analyse the changes on gross motor development after five (T5), ten (T10) and 30 (T30) months of swimming or soccer practice. The study sample consists of 33 preschoolaged boys (4.8 $\pm 0.5 \mathrm{yrs}$.): 11 soccer practitioners; 11 swimming practitioners; 11 controls (no previous involvement in sports). The Test of Gross Motor Development-Second Edition was used to assess common gross motor skills (locomotion, object control skills). Both experimental groups improved significantly in their gross motor quotient and the standard scores for locomotion and object control skills between T5 and T10. At T10, all soccer practitioners have already reached the maximum descriptive rating for the gross motor quotient. Between T10 and T30, swimming practitioners were able to improve the standard scores for object control skills. Main results showed a positive impact of swimming and soccer participation in motor proficiency.

Keywords: Swimming; soccer; motor development; childhood.
\end{abstract}

\section{INTRODUCTION}

Childhood is a key phase in the lifetime of a human being for the development of physical skills and fundamental psychomotor acquisition, which will allow, further on, the acquisition of a set of skills to influence the development of more complex motor skills (Gabbard, 2000). The gross motor development is the qualitative and quantitative progress in the motor skills, during lifetime (Gallahue \& Ozmun, 2005). The life experience of children and the stimulation they have received represent the baseline for the acquisition of more specific and critical motor skills for the different sport activities (Clark \& Metcalfe, 2002; Hands, Larkin, Parker, Straker, \& Perry, 2009; Lubans, Morgan, Cliff, Barnett, \& Okely, 2010). The phase between three and ten years old is considered the critical period in the path of gross motor development and, after that, there is a period of maturity to the acquired motor skills. Gallahue and Ozmun (2005) state that the inexistence of a rich and diverse experience of physical movements may compromise the learning of perceptive, motor and cognitive skills.

During childhood, several important development changes take place, being well established the positive influence of physical activity for a healthy growth (Boreham \& Riddoch, 2001; Eisenmann, 2003; Malina, 2007; Steele, Brage, Corder, Wareham \& Ekelund, 2008). Motor proficiency has been related with subsequent physical activity (Barnett, Van Beurden, Morgan, Brooks, \& Beard, 2009; Kambas et al., 2012). Physical activity leads to the development of fundamental motor skills (FMS) (Smith et al., 2014), including in children with coordinative difficulties (Kane \& Staples, 2014). Therefore, the literature seems to assume the existence of a strong synergistic relationship between physical activity and motor development. In this particular context, it should

\footnotetext{
Manuscript received at October $18^{\text {th }} 2015$; Accepted at December $20^{\text {th }} 2016$

${ }^{1}$ Department of Sport Sciences, University of Beira Interior, Portugal

${ }^{2}$ Research Centre for Sport, Health and Human Development (CIDESD), Portugal;

${ }^{3}$ Department of Sport and Rehabilitation Sciences, University of Liège, Belgium

${ }^{4}$ Department of Sports Sciences, Exercise and Health, University of Trás-os-Montes e Alto Douro, Portugal

${ }^{5}$ CICS-UBI Health Sciences Investigation Center, University of Beira Interior;

* Corresponding author: Departamento de Desporto da UBI, Convento de Santo António, 6201-001 Covilhã,

Portugal.E-mail: amcosta@ubi.pt
} 
be also noted that the development towards specialized motor proficiency depends on relevant previous motor experiences in a safe ageappropriate, stimulating environment (Gallahue \& Ozmun, 2005; Magill, 2000). Thus, low or inadequate motor stimulation would affect not only the child's motor development (Gallahue \& Ozmun, 2005; Williams et al., 2008), but also his cognitive, affective and social state (Busseri, Rose-Krasnor, Willoughby, \& Chalmers, 2006; Sibley \& Etnier, 2003). Likewise, it is assumed that poor gross motor development will inhibit children from regular physical activities (e.g., Stodden et al., 2008; Williams et al., 2008). In the long run, this may also determine a greater likelihood of becoming sedentary in adulthood (Huotari, Nupponen, Mikkelsson, Laakso, \& Kujala, 2011). For that reason, we should consider the assumption that childhood is not only a critical period for the acquisition of fundamental motor skills, but also to ensure lifelong participation in sport (Barnett et al., 2009; Stodden et al., 2008).

The period between five and ten years of age exhibits considerable improvement in general motor coordination, allowing the achievement of increasingly complex movements (Gallahue \& Ozmun, 2005; Massa \& Ré, 2010). During this period of fast neurological development and large neural plasticity, the child is able to understand the rules of sport and is able to participate in structured programs of sport initiation (Ré, 2011). However, little is known about the effects of organized sports practice on gross motor development. This is an important gap in the literature because several children don't benefit from a structured sport practice at school, especially during the preschool. In fact, sport participation during childhood (especially for the younger ages) result quite often by the initiative of the child and his/her family. In this context, swimming and soccer are at the top of the list of most popular sports in several countries.

Despite the fact that aquatic programs can differ (Jorgensen, 2012), the teaching methodology usually seeks to introduce children to basic aquatic skills (Gallahue \& Ozmun, 2005). Games and several other fun activities are often used as an appropriate methodological resource to achieve aquatic readiness (Rocha, Marinho, Ferreira, \& Costa, 2014). However, studies about the effectiveness of aquatic interventions on gross motor development are scarce. Water sports appear to provide important stimulation of body perception, inducing a positive effect on abilities associated with apprehension and balance (Sigmundsson \& Hopkins, 2010). A recent study suggested that children with prior participation in swimming programs (within the educational context) demonstrate an optimized motor development, on several gross motor skill tests, but particularly on object control skills (Martins, Silva, Marinho, \& Costa, 2015).

Regarding soccer, the pedagogical intervention improves the development of individual skills (e.g., passing, dribbling, shooting and ball control), but also team effectiveness. Young players are encouraged to recognize the different game variables (e.g.: opponents, field and goalpost dimensions) and to assume a tactical collective behavior (Costa, Garganta, Greco, Mesquita \& Maia, 2011). For that reason, contemporary soccer teaching models are supported in tactical principles (Holt, Strean, \& Bengoechea, 2002). However, not enough is known about the effectiveness of the specific measures of soccer adopted in improving gross motor development. Most studies has sought to determine the effects of community and school physical activities influence on children's motor skills (e.g., Erceg, Zagorac, \& Katić, 2008). To the extent of our knowledge, only one study focused on the effects of specific (extracurricular) soccer training programs on fundamental motor skills proficiency of children (Salaj, Krmpotic \& Stamenkovic, 2016). The authors of this observational study reported that preschool children enrolled in organized exercising programs (soccer or rhythmic gymnastics) tend to achieve higher overall motor development scores than children that do not exercise additionally. It seems important to obtain a longitudinal perspective about the impact of specific sport interventions on motor proficiency particularly because some children unfortunately never benefit from any kind of 
structured physical activity (physical education at school).

Thus, this paper aims to describe the longitudinal changes in the gross motor development after five, ten and thirty months of swimming or soccer practice. We expect that both sports interventions will play a catalytic role in gross motor development. We anticipate differences in the level of acquisition and degree of mastery of some fundamental motor skills.

\section{METHOD}

\section{Participants}

This research used a convenience sample of young children that were available to participate in this study and who had a known history of swimming or soccer participation. The study sample consisted of 33 preschool-aged boys $(4.8 \pm 0.5$ yrs.), all residents on the metropolitan area of Lisbon (Portugal). At baseline, the following three groups were considered: 11 children $(5.3 \pm 0.2$ yrs. $)$ with no previous involvement in sports or any kind of structured physical activity (control group); 11 children (4.6 \pm 0.4 yrs.) involved in swimming classes at a beginner level, with five months of practice (swimming group); 11 children (4.8 \pm 0.5 yrs.) involved in soccer classes at a beginner level, with five months of practice (soccer group). All physical or psychological diseases that may have precluded ability to perform the requested training exercises and testing were considered exclusion criteria.

Children were assessed in three moments: at baseline, with 5 months of previous of swimming or soccer practice (T5); 5 months after baseline, with 10 months of swimming or soccer practice (T10); 25 months after baseline, with 30 months of swimming or soccer practice (T30). The longitudinal nature of this research did not allow an evaluation of the control group after the T10 moment; from this period onwards, most of the children included in this group started practicing sports. For obvious ethical reasons, researchers did not inhibit children and/or guardians from being involved in sport. Additionally we could not make any follow-up assessment beyond 30 months of practice because many children began to engage in other sporting activities.
All experimental procedures and protocols were conform to the Declaration of Helsinki and were approved in advance by the Data Protection Authority in Portugal, by the managers of local swimming and soccer schools involved in this study and by the Ethics Committee of the Health Sciences Faculty of the University of Beira Interior. Data confidentiality was guaranteed as well as participant's anonymity.

\section{Instruments and procedures}

Gross motor development assessment

The "test of gross motor development 2" (TGMD-2) [Ulrich, 2000] was used to assess children's competence of fundamental motor skills in three distinct moments: after five (T5), ten (T10) and thirty (T30) months of sports practice. The TGMD-2 is a norm-referenced measure with a good psychometric quality to assess gross motor skills that develop early in life (Ulrich, 2000). It has been used by several researchers in different countries, including for longitudinal follow up (Cliff, Okely, Smith, \& McKeen, 2009; Cliff, Wilson, Okely, Mickle, \& Steele, 2007; Westendorp et al., 2014). It assesses twelve fundamental motor skills typically taught in physical education to children aged from three to ten years old (Wiart \& Darrah, 2001). Skills are divided into two subtests: Locomotion (run, gallop, hop, leap, horizontal jump, skip, and slide) and Object Control (twohanded strike, stationary bounce, catch, kick and the overhand throw). Each skill defined by the TGMD-2 consists of components that together constitute mature performance of that skill.

After a standard warm-up, each skill was performed three times and measured with three to four observable criteria based upon typical movement patterns identified from motor development literature and suggested by Ulrich (2000). Each criterion was rated as zero (the criterion is observed on fewer than two of the three trials) or one (criterion is observable on at least two of the three trials). The highest total raw score for both subtests is 48 . Subtest raw scores were then converted to standard scores (ranging between one and 20) for both subtests, considering the child's age at the time. Subtest standard scores (locomotion and objected 
control) are then summed and converted to calculate each child's gross motor development quotient.

As proposed by the author (Ulrich, 2000), all participants repeated the TGMD-2 one week later (retest) in T5, T10 and in T30. All evaluations were conducted by two researchers familiar with the TGMD-2 battery, including the evaluation criteria for each fundamental motor skill. Several training sessions were performed in our laboratory. One small pilot study was also conducted using a restricted sample of five children ( $4.9 \pm 0.5$ years), not considered in the analysis. These five children were evaluated twice in a weekly timeframe. The intra-class correlation coefficients (as a measure of reliability) were very high for all measured skills (ranged from 0.80 to 1.00).

All assessments were recorded on video (Sony camera, HDR-CX115 model) that was used only for this study. The two observers analysed the images obtained and reviewed the individual performance for each motor skill, according to the proposed criteria. Then, it was given an opportunity to discuss each performance and the respective score.

Tests and retest were applied effectively in $\mathrm{T} 5$, $\mathrm{T} 10$ and T30, always under the same conditions (outdoor sport field), at the same time of day and with similar weather conditions (without rain, light breeze and on a mild air temperature). Participants wore shorts and t-shirts.

\section{Swimming and soccer practice}

Training sessions for swimming and soccer occurred at the same time, twice a week (between $6 \mathrm{~h} 00$ and $6 \mathrm{~h} 45 \mathrm{pm})$. In both sports the intervention program was elementary, following mostly a mixed pedagogical concept using games to incite children to engage into learning activities but also some individual analytical motor tasks.

Swimming lessons were carried out in deep pool (plus than $1.30 \mathrm{~m}$ ) with a water temperature of $31.5^{\circ} \mathrm{C}$ (the air temperature was $29 \pm 1^{\circ} \mathrm{C}$ and the relative humidity was $65 \%$ ). The aquatic program aimed to improve children's aquatic readiness by teaching basic aquatic fundamental skills. At the beginning all children were in a state of total inaptness to the aquatic environment with no ability to perform intended propelling actions. The pedagogical intervention was based on Langendorfer and Bruya (1995) and Canossa, Fernandes, Carmo, Andrade and Soares (2007). The following aquatic motor skills were developed: water entry; water orientation and adjustment at vertical position; breath control immersion of the face and eye opening; horizontal buoyancy; body position at ventral gliding; body position at dorsal gliding; body position at longitudinal rotation in gliding; body position at front and back somersaults; leg kick with breath control at ventral body position, with flutter boards and without any flutter device; leg kick with breath control at dorsal body position with flutter boards and without any flutter device; feetfirst and head-first entry; autonomous in deep pool (legs and arms displacement); vertical buoyancy at deep water and deep-water immersion.

The soccer practice was conducted in the outdoor school sports field with synthetic grass. The sessions were planned following a coherent pedagogical approach with the latest models of soccer teaching (e.g., Bunker \& Thorpe, 1982; Costa et al., 2011; Holt et al., 2002). The soccer training program sought to develop three major capabilities: the ability to select appropriate solutions before different game problems (decision making); the ability to perform effectively (technical training to enhance dribbling, passing, shooting, finishing and also the weak foot for youth soccer players) and the ability to play as a team (communicate and cooperate). Hence, children's specific technical skills were developed (mastery of body movement with/without the ball) but also their tactical awareness.

\section{Data Analysis}

Descriptive statistics were the mean and standard deviation. The Kolmogorov-Smirnov test was used to evaluate the normality of the distribution of the variables. The Mann-Whitney $\mathrm{U}$ test was used to compare differences between two independent groups. Kruskal-Walli's test was used for multiple group comparisons. The intragroup difference between assessment moments 
was tested with the Wilcoxon signed-rank-sum test. The analyses were adjusted using the Holm's sequential Bonferroni correction (Holm, 1979); according to this sequential rejective multiple test procedure, the adjusted $\mathrm{p}$-value for $\mathrm{n}$ paired comparisons is:

$$
\text { pBonferroni } \mathrm{C}=(\mathrm{C}-\mathrm{i}+1) \times \mathrm{p}-\text { value; }
$$

where $\mathrm{C}$ correspond to number of comparisons and $\mathrm{i}$ rank of the pair in terms of degree of significance. The nonparametric effect size was obtained from the following equation (Rosenthal, 1994):

$$
r=\frac{Z}{\sqrt{N}}
$$

where $\mathrm{Z}$ is the $\mathrm{Z}$ statistic, $\mathrm{N}$ the sample size $(r<0.1$ was considered a trivial effect, $0.1 \leq r<0.3$ small effect, $0.3 \leq r \leq 0.5$ moderate and $r>0.5$ large effect). The intra-class correlation coefficient (ICC) was used as a measure of consistency of ratings over time. To establish statistical significance, a $\mathrm{p} \leq .05$ criterion was used. All data were analysed using the software SPSS 22.0.

Table 1

Raw and standard scores (mean \pm standard deviation) of the Locomotor and Object Control subtests and the respective TGMD-

\begin{tabular}{|c|c|c|c|c|c|c|}
\hline & & \multicolumn{2}{|c|}{ Locomotor subtest } & \multicolumn{2}{|c|}{ Object Control Subtest } & \multirow[t]{2}{*}{$\begin{array}{l}\text { TGMD-2 } \\
\text { quotient }\end{array}$} \\
\hline & & Raw score & Standard score & Raw score & Standard score & \\
\hline \multirow[b]{3}{*}{ 号 } & T5 & $32.00 \pm 6.02$ & $9.45 \pm 2.16$ & $31.81 \pm 6.79^{a}$ & $10.00 \pm 2.41^{\mathrm{a}}$ & $98.36 \pm 11.33^{a}$ \\
\hline & $\mathrm{T} 10$ & $35.36 \pm 8.33^{\mathrm{a}}$ & $10.45 \pm 3,39^{a}$ & $32.18 \pm 5.33^{a}$ & $9.27 \pm 1.85^{\mathrm{a}}$ & $99.18 \pm 12.59^{a}$ \\
\hline & 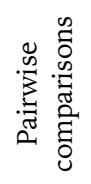 & $\begin{array}{c}\mathrm{T} 5=\mathrm{T} 10 \\
(\mathrm{p}=0.091, \mathrm{r}=.51 ; \mathrm{ICC}=.710)\end{array}$ & $\begin{array}{c}\mathrm{T} 5=\mathrm{T} 10 \\
(\mathrm{p}=.231, \mathrm{r}=0.36 ; \mathrm{ICC}=.579)\end{array}$ & $\begin{array}{c}\mathrm{T} 5=\mathrm{T} 10 \\
(\mathrm{p}=.0538, \mathrm{r}=.19 ; \mathrm{ICC}=.324)\end{array}$ & $\begin{array}{c}\mathrm{T} 5=\mathrm{T} 10 \\
(\mathrm{p}=.667, \mathrm{r}=.13 ; \mathrm{ICC}=.304)\end{array}$ & $\begin{array}{c}\mathrm{T} 5=\mathrm{T} 1 \\
(\mathrm{p}=1,000, \mathrm{r}=0 ; \mathrm{ICC}=.554)\end{array}$ \\
\hline \multirow{2}{*}{ 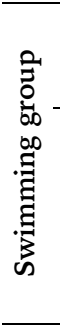 } & $\begin{array}{l}\text { T5 } \\
\text { T10 } \\
\text { T30 } \\
\end{array}$ & $\begin{array}{c}29.91 \pm 12.87 \\
40.00 \pm 8.67^{\mathrm{a}, \mathrm{b}} \\
46.36 \pm 1.96\end{array}$ & $\begin{array}{c}10.73 \pm 4.38 \\
14.09 \pm 4.25^{\mathrm{a}, \mathrm{b}} \\
14.73 \pm 1.68\end{array}$ & $\begin{array}{l}26.09 \pm 9.97^{\mathrm{a}, \mathrm{b}} \\
36.18 \pm 6.21^{\mathrm{a}, \mathrm{b}} \\
46.18 \pm 2.08^{\mathrm{a}, \mathrm{b}}\end{array}$ & $\begin{array}{c}9.91 \pm 3.27^{\mathrm{a}, \mathrm{b}} \\
12.18 \pm 2.14^{\mathrm{a}, \mathrm{b}} \\
13.55 \pm 1.57^{\mathrm{a}, \mathrm{b}}\end{array}$ & $\begin{array}{c}101.91 \pm 19.82^{\mathrm{a}} \\
118.82 \pm 15.48^{\mathrm{a}, \mathrm{b}} \\
124.81 \pm 7.83^{\mathrm{a}, \mathrm{b}} \\
\end{array}$ \\
\hline & 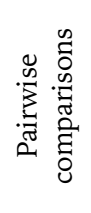 & $\begin{array}{c}\mathrm{T} 5<\mathrm{T} 10 \\
(\mathrm{p}=.008, \mathrm{r}=.86 ; \mathrm{ICC}=.810) \\
\mathrm{T} 10<\mathrm{T} 30 \\
(\mathrm{p}=.007, \mathrm{r}=.81 ; \mathrm{ICC}=.293) \\
\mathrm{T} 5<\mathrm{T} 30 \\
(\mathrm{p}=.009, \mathrm{r}=.89 ; \mathrm{ICC}=.229)\end{array}$ & $\begin{array}{c}\mathrm{T} 5<\mathrm{T} 10 \\
(\mathrm{p}=.021, \mathrm{r}=.81 ; \mathrm{ICC}=.827) \\
\mathrm{T} 10=\mathrm{T} 30 \\
(\mathrm{p}=.753, \mathrm{r}=.09 ; \mathrm{ICC}=.385) \\
\mathrm{T} 5<\mathrm{T} 30 \\
(\mathrm{p}=.018, \mathrm{r}=.79 ; \mathrm{ICC}=.337)\end{array}$ & $\begin{array}{c}\mathrm{T} 5<\mathrm{T} 10 \\
(\mathrm{p}=.004, \mathrm{r}=.86 ; \mathrm{ICC}=.700) \\
\mathrm{T} 10<\mathrm{T} 30 \\
(\mathrm{p}=.009, \mathrm{r}=.89 ; \mathrm{ICC}=.557) \\
\mathrm{T} 5<\mathrm{T} 30 \\
(\mathrm{p}=.009, \mathrm{r}=.89 ; \mathrm{ICC}=.293)\end{array}$ & $\begin{array}{c}\mathrm{T} 5<\mathrm{T} 10 \\
(\mathrm{p}=.018, \mathrm{r}=79 ; \mathrm{ICC}=.737) \\
\mathrm{T} 10<\mathrm{T} 30 \\
(\mathrm{p}=.014, \mathrm{r}=.74 ; \mathrm{ICC}=.765) \\
\mathrm{T} 5<\mathrm{T} 30 \\
(\mathrm{p}=.021, \mathrm{r}=.82 ; \mathrm{ICC}=.586)\end{array}$ & $\begin{array}{c}\mathrm{T} 5<\mathrm{T} 10 \\
(\mathrm{p}=.012, \mathrm{r}=.83 ; \mathrm{ICC}=.817) \\
\mathrm{T} 10=\mathrm{T} 30 \\
(\mathrm{p}=.154, \mathrm{r}=.43 ; \mathrm{ICC}=.480) \\
\mathrm{T} 5<\mathrm{T} 30 \\
(\mathrm{p}=.015, \mathrm{r}=.85 ; \mathrm{ICC}=.393)\end{array}$ \\
\hline \multirow[b]{2}{*}{ 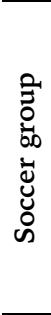 } & $\begin{array}{c}\text { T5 } \\
\text { T10 } \\
\text { T30 }\end{array}$ & $\begin{array}{c}34.09 \pm 7.27 \\
46.73 \pm 3.13^{\mathrm{a}, \mathrm{b}} \\
48.00 \pm .00\end{array}$ & $\begin{array}{c}12.45 \pm 2.98 \\
18.45 \pm 2.21^{\mathrm{a}, \mathrm{b}} \\
16.00 \pm 1.26\end{array}$ & $\begin{array}{l}36.55 \pm 4.08^{a, b} \\
45.82 \pm 1.40^{a, b} \\
48.00 \pm .000^{a, b}\end{array}$ & $\begin{array}{l}13.36 \pm 1.63^{\mathrm{a}, \mathrm{b}} \\
16.45 \pm 1.29^{\mathrm{a}, \mathrm{b}} \\
15.18 \pm 0.98^{\mathrm{a}, \mathrm{b}}\end{array}$ & $\begin{array}{l}117.45 \pm 11.60^{\mathrm{a}} \\
144.73 \pm 6.36^{\mathrm{a}, \mathrm{b}} \\
133.55 \pm 6.67^{\mathrm{a}, \mathrm{b}}\end{array}$ \\
\hline & 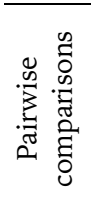 & $\begin{array}{c}\mathrm{T} 5<\mathrm{T} 10 \\
(\mathrm{p}=.009, \mathrm{r}=.89 ; \mathrm{ICC}=.195) \\
\mathrm{T} 10=\mathrm{T} 30 \\
(\mathrm{p}=.180, \mathrm{r}=.40, ¥) \\
\mathrm{T} 5<\mathrm{T} 30 \\
(\mathrm{p}=.009, \mathrm{r}=.89, ¥)\end{array}$ & $\begin{array}{c}\mathrm{T} 5<\mathrm{T} 10 \\
(\mathrm{p}=.009, \mathrm{r}=.89 ; \mathrm{ICC}=.331) \\
\mathrm{T} 10>\mathrm{T} 30 \\
(\mathrm{p}=.033, \mathrm{r}=.64 ; \mathrm{ICC}=.185) \\
\mathrm{T} 5<\mathrm{T} 30 \\
(\mathrm{p}=.024, \mathrm{r}=.76 ; \mathrm{ICC}=.115)\end{array}$ & $\begin{array}{c}\mathrm{T} 5<\mathrm{T} 10 \\
(\mathrm{p}=.009, \mathrm{r}=.89 ; \mathrm{ICC}=.312) \\
\mathrm{T} 10<\mathrm{T} 30 \\
(\mathrm{p}=.006, \mathrm{r}=.83, ¥) \\
\mathrm{T} 5<\mathrm{T} 30 \\
(\mathrm{p}=.009, \mathrm{r}=.89, ¥)\end{array}$ & $\begin{array}{c}\mathrm{T} 5<\mathrm{T} 10 \\
(\mathrm{p}=.009, \mathrm{r}=.90 ; \mathrm{ICC}=.748) \\
\mathrm{T} 10>\mathrm{T} 30 \\
(\mathrm{p}=.011, \mathrm{r}=.77 ; \mathrm{ICC}=.538) \\
\mathrm{T} 5<\mathrm{T} 30 \\
(\mathrm{p}=.008, \mathrm{r}=.86 ; \mathrm{ICC}=.513)\end{array}$ & $\begin{array}{c}\mathrm{T} 5<\mathrm{T} 10 \\
(\mathrm{p}=.009, \mathrm{r}=.89 ; \mathrm{ICC}=.533) \\
\mathrm{T} 10>\mathrm{T} 30 \\
(\mathrm{p}=.008, \mathrm{r}=.87 ; \mathrm{ICC}=.575) \\
\mathrm{T} 5<\mathrm{T} 30 \\
(\mathrm{p}=.008, \mathrm{r}=.81 ; \mathrm{ICC}=.335)\end{array}$ \\
\hline
\end{tabular}
2 quotient for all groups and assessment moments.

Note. All p-values were corrected according to the Holm-Bonferroni procedure. $(a)=$ significant $(p<0.05)$ differences in motor proficiency between all groups; $(b)=$ significant differences $(p<0.05)$ in motor proficiency between swimmers and soccer players; $(¥)=$ ICC was not calculated because one of the component variable has zero variance and is removed from the scale.

\section{RESULTS}

Table 1 presents the subtests scores (raw and standard scores) and the gross motor quotient for all groups and assessment moments separately. Both experimental groups showed significant improvements between $\mathrm{T} 5$ and $\mathrm{T} 10$ in the gross motor quotient and in the standard scores of both subtests. The control group showed no significant improvement in this regard. Significant differences were found $(\mathrm{p}<0.05)$ between groups at T5, T10 and T30 for the object control standard score and also for the gross motor quotient. Inter-group differences were also found between swimmers and soccer practitioners for the locomotor standard score (at T10, $\mathrm{p}=0.009, \mathrm{r}=0.79$ ), for the object control 
standard score (T5, $\mathrm{p}=0.022, \mathrm{r}=0.69 ; \mathrm{T} 10$, $\mathrm{p}=0.000, \mathrm{r}=1.11$; $\mathrm{T} 30, \mathrm{p}=0.014, \mathrm{r}=0.74)$ and for the gross motor quotient (T10, $\mathrm{p}=0.001, \mathrm{r}=1.04$; $\mathrm{T} 30, \mathrm{p}=0.022, \mathrm{r}=0.69)$.
One can note in table 2 a distribution of participants that tends to higher levels of motor development over time. In fact, at T10 and T30, most participants (swimmers and soccer practitioners) were ranked above average levels.

Table 2

Distribution of descriptive ratings for the gross motor quotient.

\begin{tabular}{|c|c|c|c|c|c|c|c|}
\hline & & $\begin{array}{l}\text { Poor } \\
(70-79)\end{array}$ & $\begin{array}{c}\text { Below } \\
\text { Average } \\
(80-89)\end{array}$ & $\begin{array}{l}\text { Average } \\
(90-110)\end{array}$ & $\begin{array}{c}\text { Above average } \\
(111-120)\end{array}$ & $\begin{array}{l}\text { Superior } \\
(121-130)\end{array}$ & $\begin{array}{c}\text { Very superior } \\
(>130)\end{array}$ \\
\hline \multirow{5}{*}{ 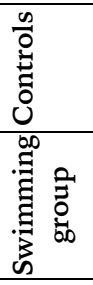 } & T5 & $1(9.1 \%)$ & $1(9.1 \%)$ & $7(63.6 \%)$ & $2(18.2 \%)$ & $0(0.0 \%)$ & $0(0.0 \%)$ \\
\hline & $\mathrm{T} 10$ & $1(9.1 \%)$ & $2(18.2 \%)$ & $6(54.5 \%)$ & $2(18.2 \%)$ & $0(0.0 \%)$ & $0(0.0 \%)$ \\
\hline & T5 & $2(18.2 \%)$ & $2(18.2 \%)$ & $4(36.4 \%)$ & $0(0.0 \%)$ & $3(27.3 \%)$ & $0(0.0 \%)$ \\
\hline & $\mathrm{T} 10$ & $0(0.0 \%)$ & $0(0.0 \%)$ & $5(45.5 \%)$ & $1(9.1 \%)$ & $3(27.3 \%)$ & $2(18.2 \%)$ \\
\hline & $\mathrm{T} 30$ & $0(0.0 \%)$ & $0(0.0 \%)$ & $0(0.0 \%)$ & $4(36.4 \%)$ & $5(45.5 \%)$ & $2(18.2 \%)$ \\
\hline \multirow{3}{*}{ 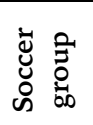 } & T5 & $0(0.0 \%)$ & $0(0.0 \%)$ & $3(27.3 \%)$ & $2(18.2 \%)$ & $5(45.5 \%)$ & $1(9.1 \%)$ \\
\hline & $\mathrm{T} 10$ & $0(0.0 \%)$ & $0(0.0 \%)$ & $0(0.0 \%)$ & $0(0.0 \%)$ & $0(0.0 \%)$ & $11(100.0 \%)$ \\
\hline & T30 & $0(0.0 \%)$ & $0(0.0 \%)$ & $0(0.0 \%)$ & $0(0.0 \%)$ & $4(36.4 \%)$ & $7(63.6 \%)$ \\
\hline
\end{tabular}

Note. Data shows the relative number of subjects (n) along with its respective percentage (\%).

Regarding the results for each locomotor fundamental skills (table 3), significant improvements $(\mathrm{p}<0.05)$ in running proficiency between T5 and T10 in control participants were identified. During this period, the soccer practitioners improved significantly their standard scores in hopping. In turn, the swimmers showed improvements $(\mathrm{p}<0.05)$ in running, galloping and hopping but not in leaping, horizontal jumping and sliding. Between T10 and T30, soccer players showed no significant improvements in these skills. Within a longer range ( $\mathrm{T} 5$ versus $\mathrm{T} 30$ ), swimmers were able to improve their motor proficiency in running, galloping and hopping. As for the soccer players, locomotor skills improved significantly $(\mathrm{p}<0.05)$ between T5 and T30 only for hopping. At $\mathrm{T} 5$, the inter-group comparison showed no significant differences between groups in these skills. However, groups differ from each other in T10 ( $\mathrm{p}<0.05)$ in almost all locomotor skills, being the group of soccer practitioners more proficient $(\mathrm{p}<0.05)$ than swimmers in hop $(\mathrm{p}$ $=0.47, \mathrm{r}=0.68)$. After 30 months of sport practice, no significant differences $(\mathrm{p}>0.05$ ) were found between both experimental groups.

Following the trend observed in the locomotor subtest, between T5 and T10 the control group did not show any significant variations in object control skills. During this period, soccer players showed proficiency increases on most evaluated fundamental skills, except in underhand roll and catch (the proficiency level in T5 for the catch skill was already maximum). However, the swimmers were able to improve their motor proficiency in striking a stationary ball, in stationary dribble and also in underhand roll. Between $\mathrm{T} 10$ and $\mathrm{T} 30$, no significant variations were identified in soccer player's motor proficiency for any object control skills, due to the high level already achieved in T10. In turn, the swimmers showed a significant evolution in almost all the skills tested during this period. In a long-term perspective (T5 vs. T30), both experimental groups showed significant improvements in most object control skills. At T5 and $\mathrm{T} 10$, significant differences $(\mathrm{p}<0.05)$ were found between groups, in most object control skills, except for striking a stationary ball (T5 and T10) and for overhand throw (T5). Actually, at T5 and T10 the group of soccer practitioners were even more proficient than swimmers in stationary dribble ( $\mathrm{T} 5, \mathrm{p}=0.021, \mathrm{r}=0.69 ; \mathrm{T} 10, \mathrm{p}=0.002$, $\mathrm{r}=0.95)$, catch $(\mathrm{T} 5, \mathrm{p}=0.002, \mathrm{r}=0.95 ; \mathrm{T} 10$, $\mathrm{p}=0.002, \mathrm{r}=0.93)$, kick (T5, $\mathrm{p}=0.010, \mathrm{r}=0.78$; $\mathrm{T} 10, \mathrm{p}=0.002, \mathrm{r}=0.92)$ and underhand roll $(\mathrm{T} 5$, $\mathrm{p}=0.001, \mathrm{r}=1.05)$. At T30, following the trend of the previous subset, no significant differences were found between the practitioners of swimming and soccer. 
Table 3

Standard scores (mean \pm standard deviation) for the Locomotor subtest

\begin{tabular}{|c|c|c|c|c|c|c|c|}
\hline & & $\begin{array}{l}\text { Run } \\
(0-8)\end{array}$ & $\begin{array}{c}\text { Gallop } \\
(0-8)\end{array}$ & $\begin{array}{l}\text { Hop } \\
(0-10)\end{array}$ & $\begin{array}{l}\text { Leap } \\
(0-6)\end{array}$ & $\begin{array}{c}\text { Horizontal } \\
\text { Jump } \\
(0-8)\end{array}$ & $\begin{array}{l}\text { Slide } \\
(0-8)\end{array}$ \\
\hline \multirow{4}{*}{$\begin{array}{l}\text { n } \\
0 \\
0 \\
0 \\
0\end{array}$} & T5 & $5.00 \pm 3.07$ & $3.73 \pm 3.10$ & $6.36 \pm 2.34$ & $5.00 \pm 1.18$ & $4.55 \pm 2.98$ & $7.36 \pm 0.92$ \\
\hline & $\mathrm{T} 10$ & $6.91 \pm 1.30^{\mathrm{a}}$ & $4.18 \pm 2.89^{\mathrm{a}}$ & $6.45 \pm 4.03^{\mathrm{a}}$ & $4.36 \pm 1.75^{\mathrm{a}}$ & $6.36 \pm 1.75$ & $7.09 \pm 1.38^{a}$ \\
\hline & Pairwise & $\mathrm{T} 5<\mathrm{T} 10$ & $\mathrm{~T} 5=\mathrm{T} 10$ & $\mathrm{~T} 5=\mathrm{T} 10$ & $\mathrm{~T} 5=\mathrm{T} 10$ & $\mathrm{~T} 5=\mathrm{T} 10$ & $\mathrm{~T} 5=\mathrm{T} 10$ \\
\hline & comparisons & $(\mathrm{p}=.039, \mathrm{r}=.62)$ & $(\mathrm{p}=.551, \mathrm{r}=.18)$ & $(\mathrm{p}=.932, \mathrm{r}=.03)$ & $(\mathrm{p}=.216, \mathrm{r}=.37)$ & $(\mathrm{p}=.105, \mathrm{r}=.49)$ & $(\mathrm{p}=.414, \mathrm{r}=.25)$ \\
\hline \multirow{7}{*}{ 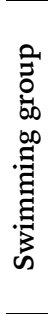 } & T5 & $5.73 \pm 1.79$ & $4.18 \pm 3.16$ & $4.27 \pm 4.15$ & $5.18 \pm 1.60$ & $4.91 \pm 3.05$ & $5.64 \pm 2.54$ \\
\hline & $\mathrm{T} 10$ & $7.82 \pm .60^{\mathrm{a}}$ & $6.73 \pm 1.85^{\mathrm{a}}$ & $6.73 \pm 3.50^{\mathrm{a}, \mathrm{b}}$ & $5.45 \pm 1.81^{\mathrm{a}}$ & $6.82 \pm 2.04$ & $6.45 \pm 2.16^{\mathrm{a}}$ \\
\hline & $\mathrm{T} 30$ & $8.00 \pm .000$ & $8.00 \pm .000$ & $9.09 \pm 1.64$ & $6.00 \pm .000$ & $7.64 \pm .81$ & $7.64 \pm .081$ \\
\hline & Pairwise & $\mathrm{T} 5<\mathrm{T} 10$ & $\mathrm{~T} 5<\mathrm{T} 10$ & $\mathrm{~T} 5<\mathrm{T} 10$ & $\mathrm{~T} 5=\mathrm{T} 10$ & $\mathrm{~T} 5=\mathrm{T} 10$ & $\mathrm{~T} 5=\mathrm{T} 10$ \\
\hline & comparisons & $\begin{array}{l}(\mathrm{p}=.018, \mathrm{r}=.82) \\
\mathrm{T} 10<\mathrm{T} 30\end{array}$ & $\begin{array}{l}(\mathrm{p}=.034, \mathrm{r}=.72) \\
\mathrm{T} 10=\mathrm{T} 30\end{array}$ & $\begin{array}{l}(\mathrm{p}=.014, \mathrm{r}=.82) \\
\mathrm{T} 10<\mathrm{T} 30\end{array}$ & $\begin{array}{l}(\mathrm{p}=.593, \mathrm{r}=.16) \\
\mathrm{T} 10=\mathrm{T} 30\end{array}$ & $\begin{array}{l}(\mathrm{p}=.051, \mathrm{r}=.72) \\
\mathrm{T} 10=\mathrm{T} 30\end{array}$ & $\begin{array}{l}(\mathrm{p}=.276, \mathrm{r}=.33) \\
\mathrm{T} 10=\mathrm{T} 30\end{array}$ \\
\hline & & $\begin{array}{c}(\mathrm{p}=.317, \mathrm{r}=.30) \\
\mathrm{T} 5<\mathrm{T} 30\end{array}$ & $\begin{array}{c}(\mathrm{p}=.059, \mathrm{r}=.57) \\
\mathrm{T} 5<\mathrm{T} 30\end{array}$ & $\begin{array}{c}(\mathrm{p}=.027, \mathrm{r}=.67) \\
\mathrm{T} 5<\mathrm{T} 30\end{array}$ & $\begin{array}{c}(\mathrm{p}=.634, \mathrm{r}=.30) \\
\mathrm{T} 5=\mathrm{T} 30\end{array}$ & $\begin{array}{c}(\mathrm{p}=.132, \mathrm{r}=.56) \\
\mathrm{T} 5=\mathrm{T} 30\end{array}$ & $\begin{array}{c}(\mathrm{p}=.082, \mathrm{r}=.62) \\
\mathrm{T} 5=\mathrm{T} 30\end{array}$ \\
\hline & & $(\mathrm{p}=.014, \mathrm{r}=.82)$ & $(\mathrm{p}=.021, \mathrm{r}=.81)$ & $(\mathrm{p}=.009, \mathrm{r}=.89)$ & $(\mathrm{p}=.306, \mathrm{r}=.49)$ & $(\mathrm{p}=.051, \mathrm{r}=.72)$ & $(\mathrm{p}=.081, \mathrm{r}=.67)$ \\
\hline \multirow{8}{*}{ 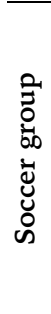 } & T5 & $4.45 \pm 3.62$ & $6.00 \pm 2.79$ & $3.55 \pm 1.04$ & $5.45 \pm 1.29$ & $7.00 \pm 1.61$ & $7.64 \pm .67$ \\
\hline & $\mathrm{T} 10$ & $8.00 \pm .000^{a}$ & $8.00 \pm .000^{a}$ & $9.45 \pm 1.29 \mathrm{a}, \mathrm{b}$ & $6.00 \pm .00^{\mathrm{a}}$ & $7.27 \pm 1.85$ & $8.00 \pm .000^{\mathrm{a}}$ \\
\hline & $\mathrm{T} 30$ & $8.00 \pm .000$ & $8.00 \pm .000$ & $8.00 \pm .000$ & $6.00 \pm 0.000$ & $8.00 \pm .000$ & $8.00 \pm .000$ \\
\hline & Pairwise & $\mathrm{T} 5=\mathrm{T} 10$ & $\mathrm{~T} 5=\mathrm{T} 10$ & $\mathrm{~T} 5<\mathrm{T} 10$ & $\mathrm{~T} 5=\mathrm{T} 10$ & $\mathrm{~T} 5=\mathrm{T} 10$ & $\mathrm{~T} 5=\mathrm{T} 10$ \\
\hline & comparisons & $(\mathrm{p}=.051, \mathrm{r}=.72)$ & $\begin{array}{l}(\mathrm{p}=.126, \mathrm{r}=.61) \\
\mathrm{T} 10=\mathrm{T} 30\end{array}$ & $(\mathrm{p}=.006, \mathrm{r}=.91)$ & $(\mathrm{p}=.540, \mathrm{r}=.40)$ & $\begin{array}{l}(\mathrm{p}=.684, \mathrm{r}=.12) \\
\mathrm{T} 10=\mathrm{T} 30\end{array}$ & $\begin{array}{l}(\mathrm{p}=.306, \mathrm{r}=.49) \\
\mathrm{T} 10=\mathrm{T} 30\end{array}$ \\
\hline & & $(\mathrm{p}=1.00, \mathrm{r}=.00)$ & $(\mathrm{p}=1.00, \mathrm{r}=.00)$ & $(\mathrm{p}=.180, \mathrm{r}=.40)$ & $(\mathrm{p}=1.00, \mathrm{r}=.00)$ & $(\mathrm{p}=.360, \mathrm{r}=.40)$ & $(\mathrm{p}=1.00, \mathrm{r}=.00)$ \\
\hline & & $\mathrm{T} 5=\mathrm{T} 30$ & $\mathrm{~T} 5=\mathrm{T} 30$ & $\mathrm{~T} 5<\mathrm{T} 30$ & $\mathrm{~T} 5=\mathrm{T} 30$ & $\mathrm{~T} 5=\mathrm{T} 30$ & $\mathrm{~T} 5=\mathrm{T} 30$ \\
\hline & & $(\mathrm{p}=.051, \mathrm{r}=.72)$ & $(\mathrm{p}=.126, \mathrm{r}=.61)$ & $(\mathrm{p}=.006, \mathrm{r}=.94)$ & $(\mathrm{p}=.540, \mathrm{r}=.40)$ & $(\mathrm{p}=.198, \mathrm{r}=.56)$ & $(\mathrm{p}=.306, \mathrm{r}=.49)$ \\
\hline
\end{tabular}

Note. All p-values were corrected according to the Holm-Bonferroni procedure. (a) = significant $(\mathrm{p}<0.05)$ differences in motor proficiency between all groups; $(b)=$ significant differences $(\mathrm{p}<0.05)$ in motor proficiency between swimmers and soccer players.

Table 4

Standard scores for the Object Control subtest

\begin{tabular}{|c|c|c|c|c|c|c|c|}
\hline & & $\begin{array}{l}\text { Striking a } \\
\text { stationary } \\
\text { ball } \\
(0-10)\end{array}$ & $\begin{array}{c}\text { Stationary } \\
\text { dribble } \\
(0-8)\end{array}$ & $\begin{array}{l}\text { Catch } \\
(0-6)\end{array}$ & $\begin{array}{l}\text { Kick } \\
(0-8)\end{array}$ & $\begin{array}{c}\text { Overhand } \\
\text { throw } \\
(0-8)\end{array}$ & $\begin{array}{c}\text { Underhand } \\
\text { roll } \\
(0-8)\end{array}$ \\
\hline \multirow{4}{*}{$\begin{array}{l}0 \\
0 \\
0 \\
0 \\
0 \\
0\end{array}$} & T5 & $7.09 \pm 2.59$ & $4.55 \pm 3.24^{a}$ & $4.36 \pm 1.96^{\mathrm{a}}$ & $6.73 \pm 2.05^{\mathrm{a}}$ & $5.82 \pm 2.75$ & $3.27 \pm 1.85^{\mathrm{a}}$ \\
\hline & $\mathrm{T} 10$ & $7.45 \pm 3.36$ & $3.82 \pm 3.40^{\mathrm{a}}$ & $4.27 \pm 1.10^{\mathrm{a}}$ & $7.555 \pm 0.82^{\mathrm{a}}$ & $4.55 \pm 2.84^{\mathrm{a}}$ & $4.55 \pm 1.81^{\mathrm{a}}$ \\
\hline & Pairwise & $\mathrm{T} 5=\mathrm{T} 10$ & $\mathrm{~T} 5=\mathrm{T} 10$ & $\mathrm{~T} 5=\mathrm{T} 10$ & $\mathrm{~T} 5=\mathrm{T} 10$ & $\mathrm{~T} 5=\mathrm{T} 10$ & $\mathrm{~T} 5=\mathrm{T} 10$ \\
\hline & comparisons & $(p=.722, r .11)$ & $(\mathrm{p}=.496, \mathrm{r}=.21)$ & $(\mathrm{p}=.829, \mathrm{r}=.07)$ & $(\mathrm{p}=.279, \mathrm{r}=.33)$ & $(\mathrm{p}=.102, \mathrm{r}=.49)$ & $(\mathrm{p}=.102, \mathrm{r}=.49)$ \\
\hline \multirow{7}{*}{ 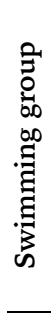 } & T5 & $7.27 \pm 3.00$ & $1.82 \pm 3.28^{\mathrm{a}, \mathrm{b}}$ & $3.27 \pm 2.00^{\mathrm{a}, \mathrm{b}}$ & $4.82 \pm 2.14^{\mathrm{a}, \mathrm{b}}$ & $5.55 \pm 2.88$ & $3.36 \pm 2.20^{\mathrm{a}, \mathrm{b}}$ \\
\hline & T10 & $9.27 \pm 1.35$ & $4.27 \pm 2.80^{\mathrm{a}, \mathrm{b}}$ & $4.09 \pm 2.21^{\mathrm{a}, \mathrm{b}}$ & $5.82 \pm 2.14^{\mathrm{a}, \mathrm{b}}$ & $7.09 \pm 1.64^{\mathrm{a}}$ & $5.64 \pm 1.50^{\mathrm{a}}$ \\
\hline & T30 & $10.00 \pm .000$ & $7.64 \pm .81$ & $5.64 \pm .81$ & $7.64 \pm .81$ & $7.64 \pm .81$ & $7.64 \pm .81$ \\
\hline & Pairwise & $\mathrm{T} 5<\mathrm{T} 10$ & $\mathrm{~T} 5<\mathrm{T} 10$ & $\mathrm{~T} 5=\mathrm{T} 10$ & $\mathrm{~T} 5=\mathrm{T} 10$ & $\mathrm{~T} 5=\mathrm{T} 10$ & $\mathrm{~T} 5<\mathrm{T} 10$ \\
\hline & comparisons & $\begin{array}{c}(\mathrm{p}=.027, \mathrm{r}=.67) \\
\mathrm{T} 10=\mathrm{T} 30\end{array}$ & $\begin{array}{l}(\mathrm{p}=.011, \mathrm{r}=.76) \\
\mathrm{T} 10<\mathrm{T} 30\end{array}$ & $\begin{array}{l}(\mathrm{p}=.230, \mathrm{r}=.36) \\
\mathrm{T} 10<\mathrm{T} 30\end{array}$ & $\begin{array}{l}(\mathrm{p}=.139, \mathrm{r}=.45) \\
\mathrm{T} 10<\mathrm{T} 30\end{array}$ & $\begin{array}{l}(\mathrm{p}=.078, \mathrm{r}=.53) \\
\mathrm{T} 10<\mathrm{T} 30\end{array}$ & $\begin{array}{l}(\mathrm{p}=.016, \mathrm{r}=.72) \\
\mathrm{T} 10<\mathrm{T} 30\end{array}$ \\
\hline & & $\begin{array}{c}(\mathrm{p}=.102, \mathrm{r}=.49) \\
\mathrm{T} 5<\mathrm{T} 30\end{array}$ & $\begin{array}{c}(\mathrm{p}=.007, \mathrm{r}=.81) \\
\mathrm{T} 5<\mathrm{T} 30\end{array}$ & $\begin{array}{c}(\mathrm{p}=.016, \mathrm{r}=.73) \\
\mathrm{T} 5<\mathrm{T} 30\end{array}$ & $\begin{array}{c}(\mathrm{p}=.016, \mathrm{r}=.73) \\
\mathrm{T} 5<\mathrm{T} 30\end{array}$ & $\begin{array}{c}(\mathrm{p}=.083, \mathrm{r}=.52) \\
\mathrm{T} 5<\mathrm{T} 30\end{array}$ & $\begin{array}{c}(\mathrm{p}=.005, \mathrm{r}=.85) \\
\mathrm{T} 5<\mathrm{T} 30\end{array}$ \\
\hline & & $(\mathrm{p}=.017, \mathrm{r}=.72)$ & $(\mathrm{p}=.006, \mathrm{r}=.83)$ & $(\mathrm{p}=.007, \mathrm{r}=.81)$ & $(\mathrm{p}=.007, \mathrm{r}=.81)$ & $(p=.026, r=.67)$ & $(\mathrm{p}=.003, \mathrm{r}=.90)$ \\
\hline \multirow{7}{*}{ 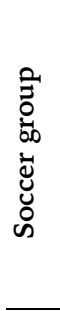 } & T5 & $5.91 \pm 1.30$ & $4.18 \pm 1.47^{\mathrm{a}, \mathrm{b}}$ & $6.00 \pm 1.18^{\mathrm{a}, \mathrm{b}}$ & $7.09 \pm .70^{\mathrm{a}, \mathrm{b}}$ & $6.45 \pm .93$ & $6.91 \pm .70^{\mathrm{a}, \mathrm{b}}$ \\
\hline & T10 & $9.45 \pm 1.29$ & $7.64 \pm .81^{\mathrm{a}, \mathrm{b}}$ & $6.00 \pm .000^{\mathrm{a}, \mathrm{b}}$ & $8.00 \pm .000^{a, b}$ & $8.00 \pm .000^{a}$ & $6.73 \pm 1.62^{a}$ \\
\hline & $\mathrm{T} 30$ & $10.00 \pm .000$ & $8.00 \pm .000$ & $6.00 \pm .000$ & $8.00 \pm .000$ & $8.00 \pm .000$ & $8.00 \pm .000$ \\
\hline & Pairwise & $\mathrm{T} 5<\mathrm{T} 10$ & $\mathrm{~T} 5<\mathrm{T} 10$ & $\mathrm{~T} 5=\mathrm{T} 10$ & $\mathrm{~T} 5<\mathrm{T} 10$ & $\mathrm{~T} 5<\mathrm{T} 10$ & $\mathrm{~T} 5=\mathrm{T} 10$ \\
\hline & comparisons & $\begin{array}{l}(\mathrm{p}=.005, \mathrm{r}=.85) \\
\mathrm{T} 10=\mathrm{T} 30\end{array}$ & $\begin{array}{c}(\mathrm{p}=.003, \mathrm{r}=.89) \\
\mathrm{T} 10=\mathrm{T} 30\end{array}$ & $\begin{array}{l}(\mathrm{p}=.942, \mathrm{r}=.02) \\
\mathrm{T} 10=\mathrm{T} 30\end{array}$ & $\begin{array}{c}(\mathrm{p}=0.008, \mathrm{r}=.80) \\
\mathrm{T} 10=\mathrm{T} 30\end{array}$ & $\begin{array}{c}(\mathrm{p}=.006, \mathrm{r}=.83) \\
\mathrm{T} 10=\mathrm{T} 30\end{array}$ & $\begin{array}{c}(\mathrm{p}=.726, \mathrm{r}=.11) \\
\mathrm{T} 10<\mathrm{T} 30\end{array}$ \\
\hline & & $\begin{array}{c}(\mathrm{p}=.180, \mathrm{r}=.40) \\
\mathrm{T} 5<\mathrm{T} 30\end{array}$ & $\begin{array}{c}(\mathrm{p}=.157, \mathrm{r}=.43) \\
\mathrm{T} 5<\mathrm{T} 30\end{array}$ & $\begin{array}{c}(\mathrm{p}=1.00, \mathrm{r}=.0) \\
\mathrm{T} 5<\mathrm{T} 30\end{array}$ & $\begin{array}{c}(\mathrm{p}=1.00, \mathrm{r}=.0) \\
\mathrm{T} 5<\mathrm{T} 30\end{array}$ & $\begin{array}{l}(\mathrm{p}=1.00, \mathrm{r}=.0) \\
\mathrm{T} 5<\mathrm{T} 30\end{array}$ & $\begin{array}{c}(\mathrm{p}=0.38, \mathrm{r}=.62) \\
\mathrm{T} 5<\mathrm{T} 30\end{array}$ \\
\hline & & $(\mathrm{p}=.003, \mathrm{r}=.90)$ & $(\mathrm{p}=.003, \mathrm{r}=.89)$ & $(\mathrm{p}=942, \mathrm{r}=.02)$ & $(\mathrm{p}=.008, \mathrm{r}=.80)$ & $(\mathrm{p}=.006, \mathrm{r}=.83)$ & $(\mathrm{p}=.006, \mathrm{r}=.83)$ \\
\hline
\end{tabular}

Note. All p-values were corrected according to the Holm-Bonferroni procedure. (a) = significant $(\mathrm{p}<0.05)$ differences in motor proficiency between all groups; $(b)=$ significant differences $(\mathrm{p}<0.05)$ in motor proficiency between swimmers and soccer players. 


\section{DISCUSSION}

This study sought to describe the longitudinal changes in motor development resulting from swimming or soccer practice in childhood. In general, our results showed a positive impact of these two sports participation in motor proficiency.

Assuming development as a dynamic system, different practice opportunities and even small differences in beginning states can amplify and lead to large individual differences in motor development (Smith \& Thelen, 2003). So, motor performance seems notably fragile and context dependent. This is an important reason why we should understand the processes by which sports activities are influenced, leading to changes on a longer time-scale.

First, we want to point out that even in a context of lack of sports participation (control group), five months (between T5 and T10) were sufficient to induce a significant impact on running ability $(\mathrm{p}=0.039)$. This seems consistent with the qualitative changes that often follows the body size growth, leading to increased levels of strength and coordination that inherently improve running performance (Haywood \& Getchell, 2004). Indeed, raw scores for five, six and seven years old children are expected to increase significantly with age (Afonso et al., 2009; Aponte, French, \& Sherrill, 1990; Ulrich, 2000). However, five months were not enough to identify significant variations in the other eleven fundamental motor skills that seem more stable over time. In fact, our results showed no significant decreases in the score means for various skills, including the standard score mean for the object control subtest (see table 4). Although we have controlled the participation in sportive activities, the circumstances and the peculiarities of the children's play weren't assessed. We recognize that this can, eventually, influence the results, just like the majority of the studies in this area. Nevertheless, it seems justified to note that the expected evolution in motor development with age isn't merely dependent on the children's growth and maturation, but is also highly influenced by environmental conditions (different practice opportunities) and suitability of the motor stimulation (e.g., Clark, 2007; Gallahue \& Ozmun, 2005).

The fast evolution of motor proficiency in the first months of sport participation (between $\mathrm{T} 5$ and T10) seems to be another important point to note in our results. Indeed, both experimental groups showed significant improvements between five and ten months of practice in locomotor and object control raw scores and also in the gross motor development quotient (see table 1). Between T10 and T30, motor proficiency is clearly less improved in both practitioners, but particularly in soccer practitioners. This is due to the fact that they have reached near maximum levels of proficiency in several fundamental motor skills at T10. In fact, we found that all soccer practitioners reach a "very superior" descriptive rate for the gross motor development in T10. The TGMD-2 battery has a high degree of reliability and low-test error (Wiart \& Darrah, 2001), but it seems to have little sensitivity to age-related improvements in participants with high or maximum motor development levels. In our opinion, this seems to be the most plausible reason for the decrease in both subtest standard scores (and in the gross motor quotient), between T10 and T30, when the raw score in most fundamental motor skills increases and reaches maximum values (or nearly that). This score limitation at the top of a scale is commonly termed "ceiling effect" (Wang, Zhang, McArdle $\&$ Salthouse, 2009).

The inter-group differences in motor proficiency are also an important outcome that should be highlighted. The results show intergroup differences that are more evident for object control skills in $\mathrm{T} 5$ and for locomotion skills in T10. This seems to mean that object control skills are more sensitive to the effects of soccer practice than actually locomotion skills, at least in these ages. In fact, in $\mathrm{T} 5$ no differences in locomotion skills were noticed between groups. Despite the difficulty in comparing these results due to lack of studies about this subject, the interpretation appears to be related to the comparability of training stimulation in interaction with biological factors (Malina \& Bouchard, 2002). 
The object control skills are clearly developed through stimulation that requires different levels of organization (e.g., two or more children to play and different forms of playing), and objects availability. In turn, locomotor skills tend to be held more trivially, spontaneously and less dependent on environment and gender differences. For that reason, the locomotion subtest raw scores are converted into standard scores, regardless of gender, and the same is not true for the object control skills.

The results regarding the continued evolution in object control proficiency in swimmers seem consistent with the data presented by Martins et al. (2015). These authors showed that previous swimming practice seems to induce a positive effect on several gross motor skills, but particularly on objects control skills. Games are used, mainly, as a natural methodological approach to teach aquatic readiness, because they combine both motivation and educational effectiveness and often the handling of several teaching materials for specific recreational purposes (Rocha et. al., 2014).

Notwithstanding, for the relevance of the present results, it should be pointed out that the current study has some limitations. First, no information about the children's play habits and/or objectively measured physical activity levels and patterns were available; these data would be very helpful in explaining the results, namely the motor proficiency increases with age. Second, no baseline data about the participant's motor proficiency, before sport practice; this would be valuable to understand the initial (first five months) effects of the practice of both sports.

As we have mentioned earlier, we have faced some constraints in the TGMD-2 evaluation program in identifying improvements of motor proficiency close or even above an advanced level. Further research attention is needed to explore possible methods of dealing with this ceiling effect in TGMD-2 longitudinal data. We also consider a very good subject for future studies the elaboration a clear set of factors to define the motor development during childhood, by combining physical aspects, contexts and opportunities of learning and stimulation programs. This will bring important guidance into the definition of school and non-school swimming programs. Given the fact that the motor development is qualitative, sequential and even cumulative, it would be very important to know better the relationship between the progress of motor proficiency in fundamental physical skills and the progress of the acquisition of specific skills in different sports, including swimming.

\section{CONCLUSION}

In conclusion, the present study showed that sport practice during childhood seems to contribute to a higher motor development. Despite of the improved motor skill competence of soccer practitioners at short and long-term, swimming practitioners show an on-going motor development particularly on object control skills.

\section{Acknowledgments:}

Nothing to declare.

\section{Conflict of Interests:}

Nothing to declare.

\section{Funding:}

Nothing to declare.

\section{REFERENCES}

Afonso, G. H., Freitas, D. L., Carmo, J. M., Lefevre, J. A., Almeida, M. J., Lopes, V. P., Neves, A. C., ..., Maia, J. A. (2009). Desempenho motor. Um estudo normativo e criterial em crianças da Região Autónoma da Madeira, Portugal. Revista Portuguesa de Ciências do Desporto, 9(2-3), 160-174.

Aponte, R., French, R., \& Sherrill, C. (1990). Motor development of Puerto Rican children: Crosscultural perspectives. Perceptual Motor Skills, 71(3Pt2), 1200-1202. Doi: 10.2466/pms.1990.71.3f.1200

Barnett, L. M., Van Beurden, E., Morgan, P. J., Brooks, L. O., \& Beard, J. R. (2009). Childhood motor skill proficiency as a predictor of adolescent physical activity. Journal of Adolescent Health, 44 (3), 252-259. Doi: 10.1016/j.jadohealth.2008.07.004

Boreham, C., \& Riddoch, C. (2001). The physical activity, fitness and health of children. Journal of sports sciences, 19(12), 915-929. Doi: 10.1080/026404101317108426 
Bunker, D. J., \& Thorpe, R. D. (1982). A model for the teaching of games in secondary schools. Bulletin of Physical Education, 18(1), 5-8.

Busseri, M. A., Rose-Krasnor, L., Willoughby, T., \& Chalmers, H. (2006). A longitudinal examination of breadth and intensity of youth activity involvement and successful development. Developmental Psychology, 42(6), 1313-1326. Doi: 10.1037/0012-1649.42.6.1313

Canossa, S., Fernandes, R., Carmo, C., Andrade, A., \& Soares, S. (2007). Ensino multidisciplinar em natação: reflexão metodológica e proposta de lista de verificação. Revista Motricidade, 4(3), 8299. Doi: $10.6063 /$ motricidade.3(4).656

Clark, J. E. (2007). On the problem of motor skill development. Journal of Physical Education, Recreation and Dance, 78(5), 39-44. Doi: 10.1080/07303084.2007.10598023

Clark, J. E., \& Metcalfe, J. S. (2002). The mountain of motor development: A metaphor. In J. E. Clark, \& J. H. Humphrey (Eds.), Motor development: Research and Reviews, (volume 2, pp. 163-190). Reston, VA: National Association for Sport and Physical Education.

Cliff, D. P., Okely, A. D., Smith, L., \& McKeen, K. (2009). Relationships between fundamental movement skills and objectively measured physical activity in pre-school children. Pediatric Exercise Science, 21 (4), 436-49.

Cliff, D. P., Wilson, A., Okely, A. D., Mickle, K. J., \& Steele, J. R. (2007). Feasibility of Shark: a physical activity skill-development program for overweight and obese children. Journal of Science and Medicine in Sport, 10(4), 263-267. Doi: 10.1016/j.jsams.2006.07.003

Costa, I. T., Garganta, J., Greco, P. J., Mesquita, I., \& Maia, J. (2011). Sistema de avaliação táctica no Futebol (FUT-SAT): Desenvolvimento e validação preliminar. Motricidade, 7(1), 69-84.

Eisenmann, J. C. (2003). Secular trends in variables associated with the metabolic syndrome of North American children and adolescents: a review and synthesis. American Journal of Human Biology, 15(6), 786-794. Doi: 10.1002/ajhb.10214

Erceg, M., Zagorac, N., \& Katić, R. (2008). The impact of football training on motor development in male children. Collegium antropologicum, 32(1), 241-247.

Gabbard, C.P. (2000). Lifelong Motor Development (3 ${ }^{\text {rd }}$ ed.). Madison Dubuque: Brown \& Benchmark.

Gallahue, D., \& Ozmun, J. (2005). Compreendendo o Desenvolvimento Motor - Bebês, Crianças, Adolescente e Adultos. Brasil: Phorte Editora.

Hands, B., Larkin, D., Parker, H., Straker, L., \& Perry, M. (2009). The relationship among physical activity, motor competence and health related fitness in 14-year-old adolescents. Scandinavian journal of medicine $\mathcal{E}$ science in sports, 19(5), 655663. Doi: 10.1111/j.1600-0838.2008.00847.x

Haywood, K., \& Getchell, N. (2004). Desenvolvimento Motor ao Longo da Vida. Porto Alegre: Artmed Editora SA.
Holm, S. (1979). A simple sequentially rejective multiple test procedure. Scandinavian Journal of Statistics, 6, 65-70.

Holt, N. L., Strean, W. B., \& Bengoechea, E. G. (2002). Expanding the teaching games for understanding model: new avenues for future research and practice. Journal of Teaching Physical Education, 21, 162-76.

Huotari, P., Nupponen, H., Mikkelsson, L., Laakso, L., \& Kujala, U. (2011). Adolescent physical fitness and activity as predictors of adulthood activity. Journal of Sports Sciences, 29(11), 1135-1141. Doi: $10.1080 / 02640414.2011 .585166$

Jorgensen, R. (2012). Under-5s Swimming as a Site for Capital Building: Supporting and Enhancing the Transitions. Australian Journal Early Child, 37 (2), 127-131.

Kambas, A., Michalopoulou, M., Fatouros, I. G., Christoforidis, C., Manthou, E., Giannakidou, D., ... \& Zimmer, R. (2012). The relationship between motor proficiency and pedometerdetermined physical activity in young children. Pediatric Exercise Science, 24(1), 34.

Kane, K. J., \& Staples, K. L. (2014). A Group Motor Skills Program for Children with Coordination Difficulties: Effect on Fundamental Movement Skills and Physical Activity Participation. Physical \& Occupational Therapy in Pediatrics, 36(1): 28-45. Doi: 10.3109/01942638.2014.978934.

Langendorfer, S., \& Bruya, L. (1995). Aquatic Readiness: Developing Water Competence in Young Children. Champaign, IL: Human Kinetics.

Lubans, D. R., Morgan, P. J., Cliff, D. P., Barnett, L. M., \& Okely, A. D. (2010). Fundamental movement skills in children and adolescents. Sports Medicine, 40(12), 1019-1035. Doi: 10.2165/11536850000000000-00000

Magill, R. A. (2000). Aprendizagem motora: conceitos e aplicações. São Paulo: Edgard Blücher.

Malina, R. (2007). Physical fitness of children and adolescents in the United States: status and secular change. Medicine and Sports Science, 50, 6790. Doi: $10.1159 / 000101076$

Malina, R.M., \& Bouchard, C. (2002). Atividade física do atleta jovem: do crescimento à maturação. São Paulo: Roca.

Martins, V., Silva, A. J., Marinho, D. A., \& Costa, A. M. (2015). Efeito da prática de natação em contexto educativo no desenvolvimento motor global de crianças do $1^{\circ}$ ciclo do ensino básico. Motricidade, 11(1): 87-97. Doi: 10.6063/motricidade.3219

Massa, M., \& Ré, A. H. (2010). Características de crescimento e desenvolvimento. In L. R. Silva (Ed.), Desempenho esportivo: Treinamento com crianças e adolescentes ( ${ }^{\text {nd }}$ ed., pp. 71-108). São Paulo: Phorte.

Ré, A. H. N. (2011). Crescimento, maturação e desenvolvimento na infância e adolescência: implicação para o esporte. Motricidade, 7(3), 5567.

Rocha, H. A., Marinho, D. A., Ferreira, S. S., \& Costa, A. M. (2014). Organização e metodologia de 
ensino da natação no $1^{\circ}$ ciclo do ensino básico em Portugal. Motricidade, 10(2), 45-59. Doi: 10.6063/motricidade.10(2).2709

Rosenthal, R. (1994). Parametric measures of effect size. In H. Cooper, \& L. V. Hedges (Eds.), The handbook of research synthesis (pp. 231-244). New York: Russell Sage Foundation.

Salaj, S., Krmpotic, M., \& Stamenkovic I. (2016). Are specific programs a threat to overall motor development of preschool children? Kinesiologia Slovenica, 22(1), 47-55.

Sibley, B. A., \& Etnier, J. L. (2003). The relationship between physical activity and cognition in children: a meta-analysis. Pediatric Exercise Science, 15(3), 243-256.

Sigmundsson, H., \& Hopkins, B. (2010). Baby swimming: exploring the effects of early intervention on subsequent motor abilities. Child: care, health and development, 36(3), 428-430. Doi: 10.1111/j.1365-2214.2009.00990.x

Smith, J. J., Eather, N., Morgan, P. J., Plotnikoff, R. C., Faigenbaum, A. D., \& Lubans, D.R. (2014). The health benefits of muscular fitness for children and adolescents: a systematic review and metaanalysis. Sports Medicine, 44(9), 1-15. Doi: 10.1007/s40279-014-0196-4.

Smith, L. B., \& Thelen, E. (2003). Development as a dynamic system. Trends in Cognitive Sciences, 7(8), 343-348. Doi: 10.1016/S1364-6613(03)00156-6

Steele, R. M., Brage, S., Corder, K., Wareham, N. J., \& Ekelund, U. (2008). Physical activity, cardiorespiratory fitness, and the metabolic syndrome in youth. Journal of Applied Physiology, 105(1), 342-351. Doi: 10.1152/japplphysiol.00072.2008

Stodden, D. F., Goodway, J. D., Langendorfer, S. J., Roberton, M. A., Rudisill, M. E., Garcia, C., \& Garcia, L. E. (2008). A developmental perspective on the role of motor skill competence in physical activity: An emergent relationship. Quest, 60(2), 290-306.

Ulrich, D. (2000). Test of Gross Motor Development Examiner's Manual (2 ed.). Austin, Texas: Pro-Ed.

Wang, L., Zhang, Z., McArdle, J. J., \& Salthouse, T. A. (2009). Investigating ceiling effects in longitudinal data analysis. Multivariate Behaviour Research, 43(3), 476-496. Doi: 10.1080/00273170802285941

Westendorp, M., Hartman, E., Houwen, S., Huijgen, B.C., Smith, J., \& Visscher, C. (2014).A longitudinal study on gross motor development in children with learning disorders. Research in Developmental Disabilities, 35(2), 357-63. Doi: 10.1016/j.ridd.2013.11.018.

Wiart, L., \& Darrah, J. (2001). Review of four tests of gross motor development. Developmental Medicine $\mathcal{E}$ Child Neurology, 43(4), 279-285.

Williams, H. G., Pfeiffer, K. A., O'Neill, J. R., Dowda, M., McIver, K. L., Brown, W. H., \& Pate, R. R. (2008). Motor skill performance and physical activity in preschool children. Obesity, 16(6), 1421-1426. Doi: 10.1038/oby.2008.214

All content of Journal Motricidade is licensed under Creative Commons, except when otherwise specified and in content retrieved from other bibliographic sources. 